\title{
PROJECTION CONSTANTS AND SPACES OF CONTINUOUS FUNCTIONS
}

BY

\author{
J. R. ISBELL AND Z. SEMADENI(1)
}

1. Theorems. A real Banach space $X$ will be called injective $\left({ }^{2}\right)$ if for every Banach space $Y$ and subspace $Y_{0}$, every linear operation $\left({ }^{3}\right) T_{0}: Y_{0} \rightarrow X$ can be extended to a linear operation $T: Y \rightarrow X$. An equivalent condition is this: for every Banach space $Z$ containing $X$ as a subspace, there exists a projection from $Z$ onto $X$. The class of injective spaces is usually denoted by $\mathfrak{P}$, and $\mathfrak{P}_{s}$ denotes the class of all spaces $X$ for which such a projection can always be found with norm $\leqq s$. If $X \in \mathfrak{P}$, then $X \in \mathfrak{P}_{s}$ for some $s \geqq 1$; the infimum $p(X)$ of such numbers $s$ is called the projection constant of $X$, and is called exact if the infimum is attained. Any Banach space $Y$ can be isometrically embedded into a space $Z$ belonging to $\mathfrak{P}_{1}$; and $Y \in \mathfrak{P}_{s}$ if and only if there exists a projection $\left({ }^{4}\right)$ $P: Z \rightarrow Y$ with $\|P\| \leqq s$ from this particular space, the best constant being independent of the choice of $Z$ and of the embedding $\left({ }^{5}\right)$. If $X \notin \mathfrak{P}$, we shall write $p(X)=\infty$.

The class $\mathfrak{P}_{1}$ is nicely characterized $\left(^{6}\right): X \in \mathfrak{P}_{1}$ if and only if $X$ is isometric to a space $C(S)$ with $S$ extremally disconnected and compact. The only known examples of spaces of $\mathfrak{P}$ are spaces isomorphic to these spaces of $\mathfrak{P}_{1}\left({ }^{7}\right)$.

Received by the editors February 27, 1962.

(1) Research supported in part by the National Science Foundation.

(2) We use the term injective to be consistent with the standard algebraic terminology. Two other terms are in use: extension property and projection property (note that this does not correspond to the algebraic term projective).

(3) In this paper, linear operations are continuous.

(4) The letter $P$ will always denote projections and if $P: Z \rightarrow Y$ is a projection, we mean $P(Z)=Y$. [37].

(5) Cf. Phillips [29], Goodner [13], Day [6, p. 94], Grünbaum [17], Nachbin [23], Sobczyk

(6) Cf. Stone [38; 39], Nakano [24], Dilworth [7], Sikorski [35, p. 257; 36], Nachbin [22], Kelley [18], Aronszajn and Panitchpakdi [2], Grünbaum [16], Grothendieck [14; 15], Lindenstrauss $[20 ; 21]$, Fullerton [11], and others. Let us mention the following characterization: $X \in \mathfrak{P}_{1}$ if and only if $X$ is isometric to the quotient space of all bounded functions satisfying the condition of Baire (cf. [19, pp. 51-60, 306-311]) on a topological space $T$ neglecting their values on sets of the first category.

(7) We do not know if there exists a space in $\mathfrak{P}$ which is not isomorphic to a space of $\mathfrak{P}_{1}$ and this problem seems to be difficult. 
Two questions are considered in this paper. The first is the projection constants of spaces $C(S)$. The simplest example of a space $C(S)$ belonging to $\mathfrak{P}$ but not to $\mathfrak{P}_{1}$ is $\left({ }^{8}\right)$

$$
X=\left\{x \in C(\beta N): x\left(t_{1}\right)=x\left(t_{2}\right)\right\},
$$

where $t_{1}$ and $t_{2}$ are two points of $\beta N \backslash N$; in other words, $S$ is obtained by identification of two nonisolated points of $\beta N$. We shall show that all the numbers $1,2,7 / 3, \cdots, 3-2 / n, \cdots, 3$ are projection constants of spaces $C(S)$ (in our examples, they are exact), but we do not know what other numbers are possible.

The theorem below establishes some necessary and some sufficient conditions concerning the possible projection constants of spaces $C(S)$ in terms of the Gleason map of $S$. A. M. Gleason [12] has proved the following theorem: For each compact $S$ there exists a unique extremally disconnected compact space $G_{S}$ with a unique irreducible $\left({ }^{9}\right)$ map $g_{S}$ from $G_{S}$ onto $S$. We shall call $G_{S}$ the Gleason space of $S$ and $g_{S}$ the Gleason map of $S$. Define the order $\iota(S)$ of the Gleason map as the least integer $n$ such that all inverse sets $g_{S}^{-1}(t), t \in S$, consist of at most $n$ points, and $\iota(S)=\infty$ if no such integer exists; $\iota(S)$ has a simple topological characterization (see Lemma 7 below).

THEOREM $1\left({ }^{10}\right)$. Let $p_{C}(S)$ be the projection constant of $C(S), S$ being a compact Hausd orff space. Then

(i) If $p_{c}(S)<3$ then the Gleason map of $S$ is of finite order; specifically

$$
\iota(S) \leqq 2\left[3-p_{C}(S)\right]^{-1}
$$

(ii) If $p_{C}(S)<2$ then $p_{C}(S)=1$.

(iii) If $\iota(S)=n$ and only a finite number of inverse sets $g_{S}^{-1}(t), t \in S$, contain more than one point, then $p_{C}(S)=3-2 / n$.

(iv) There are spaces $S$ such that $p_{c}(S)=3$.

This leaves the problem of topological characterization of spaces $S$ for which $C(S)$ is injective entirely open. Neither of the conditions $p_{c}(S)<\infty, \iota(S)<\infty$, implies the other.

(8) In the category of compact spaces and continuous mappings, extremally disconnected spaces are just those which are projective (cf. Gleason [12], Rainwater [30]), and in the category of Banach spaces, $\mathfrak{P}$ is just the class of injective spaces. If we consider $C(S)$ as a dual of $S$, then this example shows that the duals of projectives are injective but injectivity of the dual need not imply the projectivity of a compact space.

(9) A continuous map $f: A \rightarrow B$ will be called irreducible if $f(A)=B$ but $f(F) \neq B$ for any proper closed subset $F$ of $A$. Uniqueness here means that for such $G_{S}, g_{S}$ and $G_{S}^{\prime}, g_{S}^{\prime}$ there is a homeomorphism $h$ from $G_{S}^{\prime}$ onto $G_{S}$ such that $g_{S}^{\prime}=g_{S} \cdot h$.

(10) While this paper was being typed, Professor Branko Grünbaum informed us of results similar to Theorem 1 obtained simultaneously by D. Amir. Added in prooff. Amir's paper has been published in Bull. Res. Council Israel 11 (1962). 
The second topic considered in the paper is exactness of the projection constant of a Banach space. The question has been raised whether the projection constant of every space $X \in \mathfrak{P}$ is exact. The answer is no and it follows from the following theorem.

THEOREM 2. Let $S$ be a compact space containing infinitely many nonisolated points. Then there exists a Radon (= regular finite Borel signed) measure $\mu$ on $S$ such that the hyperplane

$$
X=\left\{x \in C(S): \int x d \mu=0\right\}
$$

does not admit any projection $P: C(S) \rightarrow X$ with norm $\|P\| \leqq 2$. For spaces $S$ containing only finitely many nonisolated points, there is no such measure $\left({ }^{11}\right)$.

Combine this theorem with the fact that any Banach space $Z$ can be projected onto any of its hyperplanes by projections with norm $<2+\varepsilon$, for arbitrary $\varepsilon>0\left({ }^{12}\right)$. We conclude: Every infinite dimensional space in $\mathfrak{P}_{1}$ contains a hyperplane having the nonexact projection constant 2.

B. Grünbaum has asked whether the projection constant of every finite dimensional space is exact. The answer is yes and may be stated in more general form as follows.

THEOREM 3. Let $X$ be a subspace of a Banach space $Z$ admitting a projection $P: Z \rightarrow X$ and let $X$ be isometric to the conjugate space of a Banach space $Y$. Then there exists a projection $P_{0}: Z \rightarrow X$ such that for any projection $P: Z \rightarrow X$ we have $\|P\| \geqq\left\|P_{0}\right\|$.

In particular, if $X \in \mathfrak{P}$, then application of Theorem 3 to a space $Z \in \mathfrak{P}_{1}$ containing $X$ yields the conclusion: If $X \in \mathfrak{P}$ and $X$ is a conjugate space, then the projection constant of $X$ is exact.

The paper is concluded with some remarks and problems about projection constants.

2. Proofs. Besides that in the preceding section, let us establish the following notation. $S, S_{0}, S_{1}, \cdots$ will denote always compact Hausdorff spaces and $C(S)$

(11) Let $\Gamma_{\alpha}$ be the set of ordinals smaller than or equal to $\alpha$, compact in the order topology. $\Gamma_{\omega}$ has just one limit point and $\Gamma_{\omega^{2}}$ has infinitely many of them, but $C\left(\Gamma_{\omega}\right)$ is isomorphic to $C\left(\Gamma_{\omega^{2}}\right)$. Thus, the existence of projections of norm $\|P\| \leqq 2$ onto each hyperplane is not an isomorphic invariant.

Dr. Joram Lindenstrauss recently informed us that he had proved the following: if the projection constant of a Banach space is equal to one, it is exact. At the same time, Dr. A. Pelczyński proved that for each number $s>1$ there exists a space $X$ such that $p(X)$ is equal to $s$ and is not exact.

(12) This result of Bohnenblust [4] has been discussed by Grünbaum [16] who has proved the existence of a hyperplane $H$ in $l_{1}$ which does not admit any projection $P: l_{1} \leftarrow H$ with $\|P\| \leqq 2$. Since $l_{1} \notin \mathfrak{P}$, this example did not answer the question on exactness. 
the space of real-valued continuous functions on $S, C(S \mid A)=\{x \in C(S): x(t)=0$ for $t \in A\}$. Further, $f: A \rightarrow B$ will always mean a continuous map from $A$ into $B$. Similarly, if $X, Y$ are Banach spaces, $T: X \rightarrow Y$ will mean a linear operation. $\chi_{A}$ will mean the characteristic function of $A$. Extremally disconnected may be abbreviated e.d. The $m$-product $\left(X_{1} \times X_{2} \times \cdots\right)_{m}$ of a sequence of Banach spaces means the space of all sequences $x=\left(x_{1}, x_{2}, \cdots\right)$ such that $x_{n} \in X_{n}$ and $\sup \left\|x_{n}\right\|<\infty$. Finally, $\mu(x)=\int x d \mu$ and $X_{\mu}=\{x \in C(S): \mu(x)=0\}$.

We begin with some lemmas which will be stated without proof.

LEMMA 1. Let $F$ be a closed nowhere dense subset of an e.d. compact space $E$ and let $S$ be the quotient space obtained by the identification of all points of $F$. Then $S$ is compact, $E=G_{S}$, the identification map is the Gleason map of $S$. If $F$ is infinite, $\iota(S)=\infty$.

Lemma 2. Let $F_{1}, F_{2}$ be two nowhere dense disjoint closed subsets of an e.d. compact space $E$ and let $h: F_{1} \rightarrow F_{2}$ be a homeomorphism onto. Then $S$ obtained by pairwise identification of the points $t$ and $h(t)$ is compact, $E=G_{S}$, the identification map is the Gleason map of $S$ and $\iota(S)=2$.

Lemma 3. Suppose that $0 \neq F=F \subset S$ and that there exists a simultaneous extension of the continuous functions on $F$ to continuous functions on $S$, i.e., a linear operation $T: C(F) \rightarrow C(S)$ such that $(T x)(t)=x(t)$ for $t \in F$. Then:

(i) $P_{1}(x)=T\left(\operatorname{Rest}_{F} x\right)$ is a projection from $C(S)$ onto the subspace of extended functions and $\left\|P_{1}\right\|=\|T\|$, Rest $_{F}$ being the restriction to $F$.

(ii) $P_{2}(x)=x-P_{1}(x)$ is the complementary projection from $C(S)$ onto $C(S \mid F)$ and $\left\|P_{2}\right\|=1+\|T\|$.

(iii) Given a point $t_{0} \in F, P_{3}(x)=P_{2}(x)+x\left(t_{0}\right)$ is a projection from $C(S)$ onto the subspace of functions constant on $F$ and $\left\|P_{3}\right\| \leqq 2+\|T\|$.

LEMMA 4. Let $S=F_{1} \cup \cdots \cup F_{m}$ be a decomposition of an infinite space $S$ into disjoint open sets and let $p_{1} \in F_{1}, \cdots, p_{m} \in F_{m}$. Then

$$
P(x)=x+\sum_{k=1}^{m} x\left(p_{k}\right)\left(\frac{1}{m}-\chi_{F_{k}}\right)
$$

is a projection from $C(S)$ onto $\left\{x \in C(S): x\left(p_{1}\right)=\cdots=x\left(p_{m}\right)\right\}$ and $\|P\|=3-2 m^{-1}$.

LEMMA 5. Given any sequence of Banach spaces $X_{1}, X_{2}, \cdots$, the equality $p\left[\left(X_{1} \times X_{2} \times \cdots\right)_{m}\right]=\sup _{n} p\left(X_{n}\right)$ holds (if one side is finite, so is the other). In particular, if $S_{n}$ are mutually disjoint, then $p_{C}\left[\beta\left(\bigcup S_{n}\right)\right]=\sup p_{C}\left(S_{n}\right)$.

LEMMA 6. Any open nonvoid set in $G_{S}$ contains at least one inverse set $g_{S}^{-1}(t), t \in S$.

Indeed, if it were not so, $g_{s}$ would be reducible.

LEMMA 7. Given a point $t_{0} \in S$, the following conditions are equivalent: 
(a) There are $n$ different points $u_{1}, \cdots, u_{n}$ in $G_{S}$ such that $g_{s}\left(u_{1}\right)=\cdots=$ $g_{s}\left(u_{n}\right)=t_{0}$.

(b) There are $n$ disjoint open subsets $H_{1}, \cdots, H_{n}$ of $S$ such that $t_{0} \in \bar{H}_{1} \cap \cdots \cap \bar{H}_{n}$.

Proof. Suppose (a). Let $U_{1}, \cdots, U_{n}$ be disjoint open-closed subsets of $G_{S}$ such that $U_{1} \cup \cdots \cup U_{n}=G_{S}$ and $u_{k} \in U_{k}$ for $k=1, \cdots, n$. Let $H_{k}=\{t \in S$ : $\left.g_{S}^{-1}(t) \subset U_{k}\right\}$ and $V_{k}=g_{S}^{-1}\left(H_{k}\right)$. Since $H_{k}=S \backslash \bigcup_{j \neq k} g_{S}\left(U_{j}\right), H_{k}$ and $V_{k}$ are open in $S$ and $G_{S}$, respectively, and $V_{k}$ is dense in $U_{k}$ (by Lemma 6). Obviously, $H_{1}, \cdots, H_{n}$ are disjoint and $t_{0}=g_{S}\left(u_{k}\right) \in g_{S}\left(U_{k}\right)=\bar{H}_{k}$ for $k=1, \cdots, n$.

Suppose (b). The sets $g_{S}^{-1}\left(H_{k}\right)$ are disjoint open sets in an e.d. space; hence their closures $U_{k}$ are disjoint, $k=1, \cdots, n$. Since $g_{S}\left(U_{k}\right)$ is a compact set containing $H_{k}$, there is at least one point $u_{k} \in U_{k}$ such that $g_{s}\left(u_{k}\right)=t_{0}$.

LEMMA 8. Let $M$ be a Hausdorff space containing infinitely many nonisolated points. Then $M$ has an infinite nowhere-dense subset.

Proof. If $M$ does not contain any perfect $\left({ }^{13}\right)$ subset, the set of nonisolated points of $M$ is nowhere dense. If $M$ does contain a perfect $Q, Q$ contains an infinite subset $T$ which is discrete in the relative topology; so $T$ is nowhere dense (cf. $[19$, p. 46]).

Lemma 9. Let $\mu$ be a Radon measure on $S$ such that $\|\mu\|=1$ and let some isolated point $t_{0}$ of $S$ have positive measure $\mu\left(t_{0}\right)=a_{0}>0$. Then there exists a projection $P: C(S) \rightarrow X_{\mu}$ of norm $\|P\| \leqq 2$.

Proof. If $a_{0}=\|\mu\|=1$, then there exists a projection of norm 1. Suppose $a_{0}<1$ and choose $y \in C(S)$ so that $\|y\|=1, \mu(y)>a_{0}, \mu(y)>1-a_{0}, y\left(t_{0}\right)=1$. Let $z(t)=y(t)$ for $t \neq t_{0}$ and $z\left(t_{0}\right)=1+(1-\mu(y)) a_{0}^{-1}$, and let $P(x)=x-\mu(x) z$; then $P: C(S) \rightarrow X_{\mu}$ as $\mu(z)=1$. If $x \in C(S)$ and $\|x\| \leqq 1$, then $|x(t)-\mu(x) z(t)| \leqq 2$ for $t \neq t_{0}$ and $\left|x\left(t_{0}\right)-\mu(x) z\left(t_{0}\right)\right| \leqq\left|x\left(t_{0}\right)\right|\left|1-a_{0} z\left(t_{0}\right)\right|+z\left(t_{0}\right)\|x\| \mu\left(S \backslash t_{0}\right) \leqq 1$ $-a_{0} z\left(t_{0}\right)+\left(1-a_{0}\right) z\left(t_{0}\right) \leqq 1-2 a_{0}+z\left(t_{0}\right)<2$. Thus, $\|P\| \leqq 2$.

Proof of Theorem 1. Let $\iota(S) \geqq n$ and let $P$ be any projection from $C\left(G_{S}\right)$ onto the subspace $Z$ of $C\left(G_{S}\right)$ consisting of functions constant on the inverse sets $g_{S}^{-1}(t)\left(Z\right.$ is the image of $C(S)$ in $C\left(G_{S}\right)$ ). We have to prove that $\|P\| \geqq 3-2 n^{-1}$. By Lemma 7, there are open disjoint sets $H_{1}, \cdots, H_{n}$ in $S$ and a point $t_{0} \in S$ such that $t_{0} \in \bar{H}_{1} \cap \cdots \cap \bar{H}_{n}$, the closures $U_{k}$ of $g_{S}^{-1}\left(H_{k}\right)$ are disjoint, and $G_{s}=U_{1} \cup \cdots \cup U_{n}$. Let $u_{k} \in U_{k} \cap g_{s}^{-1}\left(t_{0}\right)$. Then $x\left(u_{1}\right)=\cdots=x\left(u_{n}\right)$ for $x \in Z$.

Let $\phi_{k}=P\left(\chi_{U_{k}}\right)$ and $c_{k}=\phi_{k}\left(u_{k}\right)=\phi_{k}\left(u_{j}\right)$ for $k, j=1, \cdots, n$. Then $\Sigma \phi_{k}$ $=P\left(\sum \chi_{U_{k}}\right)=1$ whence $\Sigma c_{k}=1$. Choose $i$ so that $c_{i} \leqq 1 / n$. Let $f=2 \chi_{U_{i}}-1$ and $q=Q f$ where $Q=I-P: C\left(G_{S}\right) \rightarrow\{x: P x=0\}$. Then $q\left(u_{i}\right)=f\left(u_{i}\right)-\left(2 c_{i}-1\right)$ $=2-2 c_{i} \geqq 2-2 / n$. Given any $\varepsilon>0$, there is $w_{0} \in g_{s}^{-1}\left(H_{i}\right)$ such that $q\left(w_{0}\right)>2-2 n^{-1}-\varepsilon$. Let $h_{0}: S \rightarrow[0,2]$ be such that $h_{0}\left(g_{s}\left(w_{0}\right)\right)=2$ and

(13) By a perfect set we mean a nonempty closed dense-in-itself set. 
$h_{0}(t)=0$ for $t \in S \backslash H_{i}$, and let $h=h_{0} \cdot g_{S}$. Then $h \in Z, h(v)=0$ for $v \in G_{S} \backslash U_{i}$, $|f(v)-h(v)| \leqq 1$ for $v \in U_{i}$ and $f\left(w_{0}\right)-h\left(w_{0}\right)=-1$, whence $\|h-f\|=1$, $P(h-f)=h-f-Q(h)+Q(f)=h-f+q$ and $(h-f+q)\left(w_{0}\right)=2-1+q\left(w_{0}\right)$ $>3-2 n^{-1}-\varepsilon$. Thus, $\|P\| \geqq 3-2 n^{-1}$ and (i) has been proved.

(ii) follows from (i), because if $p_{c}(S)<2$, then $g_{s}$ must be one-to-one. (iii) follows from Lemma 4 and (iv) follows from (iii) and Lemma 5. (iv) can be also proved in another way: Take any e.d. dense-in-itself compact space $H$ (i.e., the Stone space of a complete atomless Boolean algebra). There exists $\beta N_{\alpha}$ containing $H$ as a retract (cf. Rainwater [30]); obviously, $H \subset \beta N_{\alpha} \backslash N_{\alpha}$. Consider $S$ obtained from $\beta N_{\alpha}$ by shrinking $H$ into a point. By Lemma 3, there is a projection $P_{3}$ from $C\left(\beta N_{\alpha}\right)$ onto the subspace of functions constant on $H$, of norm less than or equal to 3 . So $p_{c}(S) \leqq 3 ; p_{c}(S)<3$ would contradict (i) as the identification map $\beta N_{\alpha} \rightarrow S$ is the Gleason map (by Lemma 1).

Proof of Theorem 2. Let $S$ be compact, with infinitely many nonisolated points; let $\left\{t_{k}\right\}$ be an infinite nowhere dense set (Lemma 8) with a limit point $t_{0}$ such that $t_{0} \neq t_{k}$ for $k=1,2, \cdots$. Define $a_{0}=-1 / 2$; for $n=1,2, \cdots$, define $a_{n}$ as $1 / 2^{n+1}$. Let $\mu$ be the atomic measure $\sum_{n=0}^{n=\infty} a_{n} \varepsilon_{t_{n}}$. The norm $\|\mu\|$ is 1 ; the support $M$ of $\mu$ is the closure of $\left\{t_{k}\right\}$. Consider any $P: C(S) \rightarrow X_{\mu}$; necessarily $P(x)=x$ $-\mu(x) u$ for some $u \in C(S)$ such that $\mu(u)=1$. Since $\mu$ does not attain its norm, $\|u\|>1$; we may write $\|u\|>1 /(1-\varepsilon), \varepsilon>0$. There is an open set $V \subset S$, such that $V \cap M=\varnothing$ and either $u(t)>(1-\varepsilon)^{-1}$ on $V$ or $-u(t)>(1-\varepsilon)^{-1}$ on $V$. Take $x$ such that $\|x\|=1$ and $\mu(x)>1-\varepsilon$; let $y$ coincide with $x$ on $(S \backslash V) \cup M$, but at some point $v$ of $V$, let $y(v)=-\operatorname{sgn} u(v) \neq 0$. This can be done with $\|y\|=1$. Since $y=x$ on $M, \mu(y)>1-\varepsilon$. Then $\|P(y)\|=\|y-\mu(y) u\|>2$ as

$$
|y(v)-\mu(y) u(v)|>\left|-1-(1-\varepsilon)(1-\varepsilon)^{-1}\right|=2 .
$$

Now, suppose that $S$ has only finitely many nonisolated points. Given any Radon measure $\mu$ on $S$ with $\mu(S)=1$, we have to prove that there exists $P: C(S) \rightarrow X_{\mu}$ with $\|P\| \leqq 2$. If the measure is concentrated on nonisolated points, there exists $u \in C(S)$ such that $\|u\|=1$ and $\mu(u)=1$, whence $P(x)=x-\mu(x) u$ has the desired property; in the contrary case we apply Lemma 9 .

Proof of Theorem 3. The relation $\psi(z, y)=(T z) y$ establishes a one-to-one isometrically isomorphic correspondence from the space $B(Z, X)$ of linear operations from $Z$ to $X$ onto the space $H(Z, Y)$ of bilinear functionals on $Z \times Y$. Let $\left\{z_{\alpha}\right\}_{\alpha \in A}$ and $\left\{y_{\beta}\right\}_{\beta \in B}$ be normalized Hamel bases for $Z$ and $Y$, respectively. The sets

$$
U\left(T_{0}\right)=\bigcap_{i=1}^{n} \bigcap_{j=1}^{m}\left\{T \in B(Z, X):\left|\left(T z_{\alpha_{i}}-T_{0} z_{\alpha_{i}}\right) y_{\beta_{j}}\right|<\varepsilon\right\}
$$

with $\alpha_{1}, \cdots, \alpha_{n} \in A, \beta_{1}, \cdots, \beta_{m} \in B, \varepsilon>0$, form a basis of neighborhoods for a 
*-weak operator topology in $B(Z, X)$. The space $H_{0}$ of all (not necessarily continuous) bilinear forms on $Z \times Y$ is homeomorphic to a product of real lines, and

$$
K_{0}=\bigcap_{\alpha \in A} \bigcap_{\beta \in B}\left\{\psi \in H_{0}:\left|\psi\left(z_{\alpha}, y_{\beta}\right)\right| \leqq 1\right\}
$$

is homeomorphic to a Tichonov cube. Since the unit cell $K=\{T \in B(Z, X):\|T\|$ $\leqq 1\}$ is mapped onto a closed subset of $K_{0}, K$ is compact.

Now, if $P_{n}: Z \rightarrow X$ are projections and $\left\|P_{n}\right\| \leqq s+n^{-1}$, then any limit point $P$ of $P_{n}$ in this topology is a projection $P: Z \rightarrow X$ and $\|P\| \leqq s$.

3. Remarks and problems. We begin by showing some counterexamples.

Proposition 1. There exists $S$ such that $\iota(S)=2$ and $p_{C}(S)=\infty$.

Proof. Let $N^{\prime}, N^{\prime \prime}$ be two countable discrete spaces, $h: \beta N^{\prime} \rightarrow \beta N^{\prime \prime}$ an identification homeomorphism, let $Q=\beta N^{\prime} \cup \beta N^{\prime \prime}$ (as disjoint copies) and

$$
X=\left\{x \in C(Q): x(t)=x(h(t)) \text { for all } t \in \beta N^{\prime} \backslash N^{\prime}\right\} .
$$

Then $X$ is isometric to a space $C(S)$ and $\iota(S)=2$ by Lemma 2. On the other hand, let $(T x)(t)=x(t)$ for $t \in \beta N^{\prime}$ and $(T x)(t)=x(t)-x\left(h^{-1}(t)\right)$ for $t \in \beta N^{\prime \prime}$. Then $T$ is an isomorphism from $X$ onto $C\left(Q \mid \beta N^{\prime \prime} \backslash N^{\prime \prime}\right)$ and the last space is obviously isomorphic to the Cartesian product of $C(\beta N)$ and $C(\beta N \mid \beta N \backslash N)$. In turn, $C(\beta N \mid \beta N \backslash N)$ is isometric to the space $c_{0}$ of sequences convergent to 0 . Thus, $X \notin \mathfrak{P}$, because if $X$ were injective, so would $c_{0}$ be, contradicting a well-known theorem of Phillips [29, p. 539].

Proposition 2. There exists $S$ such that $\iota(S)=\infty$ and $p_{c}(S)=3$.

Such an example was considered in the proof of Theorem 1.

Proposition 3. There exists $S$ such that $\iota(S)=2, p_{c}(S)=2$, but infinitely many inverse sets contain more than one point.

Indeed, let $S_{0}$ be obtained by identification of two nonisolated points of $\beta N$ and let $S$ be $\beta$ of the union of $\aleph_{0}$ disjoint copies of $S_{0}$.

Another construction of spaces with $p_{C}(S)<\infty$ can be obtained by the following generalization of Lemma 4.

Proposition 4. Suppose $A_{1}, \cdots, A_{m}$ are closed, nonvoid, nowhere dense subsets of $S ; F_{1}, \cdots, F_{m}$ open disjoint sets such that $S=F_{1} \cup \cdots \cup F_{m}, A_{k} \subset F_{k}$ and suppose $T_{k}: C\left(A_{k}\right) \rightarrow C\left(F_{k}\right)$ are simultaneous extensions $(k=1, \cdots, m)$. Let $h_{i j}$ be a homeomorphism of $A_{i}$ onto $A_{j}(i, j=1, \cdots, m)$ such that $h_{i j}=h_{k j} \cdot h_{i k}$. Given a function $f \in C(S)$, let

$$
g_{k}(t)=\sum_{j=1}^{m}\left(\frac{1}{m}-\delta_{j k}\right) f\left(h_{k j}(t)\right)
$$


for $t \in A_{k}$ and $(P f)(t)=f(t)+\left(T_{k} g_{k}\right)(t)$ for $t \in F_{k}$. Then $(P f)(t)=1 / m \sum_{j} f\left(h_{i j}(t)\right)$ for $t \in A_{i}$, whence

$$
P: C(S) \rightarrow \bigcap_{i, j} \bigcap_{t \in A_{i}}\left\{x \in C(S): x\left(h_{i j}(t)\right)=x(t)\right\}
$$

and $\|P\| \leqq 1+\left(2-2 n^{-1}\right) \sup \left\|T_{k}\right\|$.

LEMMA 10. Let $\mu$ be any Radon measure on $S$, let $V$ be an infinite open subset of $S$ and let $\varepsilon>0$. There exists an open nonvoid set $W \subset V$ such that $|\mu|(W)<\varepsilon$.

Proposition 5. Let $\mu$ be a Radon measure on $S,\|\mu\|=1$, and let $M_{1}$ and $M_{2}$ be the supports of $\mu_{+}$and $\mu_{-}$, respectively. Suppose that $M_{1} \cup M_{2}$ does not contain any of the isolated points of $S$ and that $P: C(S) \rightarrow X_{\mu}$ is of the form $P(x)=x-\mu(x) u$ with $u \in C(S)$ and $\mu(u)=1$. Then $\|P\| \geqq 1+\|u\| \geqq 2$. If $M_{1} \cap M_{2} \neq \varnothing$, then $\|u\|>1$ whence $\|P\|>2$.

We omit the proofs.

Many problems have been raised on the class $\mathfrak{P}$ and some of them seem to be difficult. Besides those in $[23 ; 25]$, and others, the following ones seem to be open.

Theorem 3 gives a sufficient condition for exactness of a projection constant, but not necessary. There exist spaces in $\mathfrak{P}_{1}$ which are not isometric to any conjugate space (cf. Dixmier [9], Kelley [18], Wada [40]; the simplest example is the space of bounded Baire functions on an interval modulo null functions, but this space is isomorphic to $C(\beta N)$ (cf. $[25$, p. $223 ; 33])\left({ }^{14}\right)$.

Problem 1. Let $X \in \mathfrak{P}_{1}$. Is $X$ isomorphic to a conjugate space?

There are spaces in $\mathfrak{P}_{1}$ which are not isomorphic to any space $C\left(\beta N_{\alpha}\right), N_{\alpha}$ being a discrete space of power $\aleph_{\alpha}$ (cf. [25, p. 223]).

Proposition 6. Let $X \in \mathfrak{P}, Z \in \mathfrak{P}_{1}$ and $X \subset Z$; let $P: Z \rightarrow X,\|P\|=s>1$. Let \|\|$_{0}^{*}$ be the norm in $X$ treated as the quotient $Z / Z_{0}$ where $Z_{0}$ $=(I-P)(Z)=\{z: P z=0\}$, i.e., let $\|x\|_{0}^{*}=\inf \{\|z\|: x=P z\}$. Let \|\|$^{*}$ be the Minkowski functional of the convex hull of the union of $\left\{x \in X:\|x\|_{0}^{*} \leqq 1\right\}$ and $\{x \in Z:\|z\| \leqq 1\}$. Then \|\|$^{*}$ is a norm in $Z,\|z\|^{*} \leqq\|z\| \leqq s\|z\|^{*}$ for $z \in Z,\|x\|^{*}=\|x\|_{0}^{*}$ for $x \in X(c f .[1, p .129 ; 37, p .167])$ and $P$ treated as an operation from $\left\langle Z,\|\|^{*}\right\rangle$ onto $\left\langle X,\|\|^{*}\right\rangle$ is a projection with $\|P\|^{*}=1$.

Problem 2. Does $\left\langle Z,\|\|^{*}\right\rangle$ belong to $\mathfrak{P}_{1}$ ?

Problem 3. Characterize the compact spaces $S$ such that $p_{C}(S)<\infty$. Establish all possible values of $p_{C}(S)$.

The second part is related to the problem of estimation of the infimum of the norms of simultaneous extensions of continuous functions from a given sub-

(14) A. Pełczyński has remarked that if a space $C(S)$ is isomorphic to the conjugate space of a Banach space, then $C(S) \in \mathfrak{P}$. Indeed, by a theorem of Dixmier [8, p. 1066], for every Banach space $X$, there exists a projection of norm 1 from $X^{* * *}$ onto the canonical image of $X^{*}$ in $X^{* * *}$ and the second dual of any space $C(S)$ is in $\mathfrak{P}_{1}$. 
space $S_{0}$ of a space $S$. There are some sufficient conditions for existence of such $T: C\left(S_{0}\right) \rightarrow C(S)$ with $\|T\|=1$ (Borsuk [5], Kakutani, Dugundji [10] and others), but little is known about the case $\|T\|>1$.

Problem 4. Suppose $p_{c}(S)<\infty$. Must this projection constant be exact? Must $C(S)$ be isomorphic to a space of $\mathfrak{P}_{1}$ ?

Now, given a Banach space $X$, let $X_{s}$ be the set of all functionals $x^{* *}$ of the bidual $X^{* *}$ which are sequentially continuous with respect to the weak topology $\sigma\left(X^{*}, X\right)$, i.e., such that the condition $x_{n}^{*}(x) \rightarrow 0$ for all $x \in X$ implies $x^{* *}\left(x_{n}^{*}\right) \rightarrow 0$.

LEMMA 11. If $T^{* *}: X^{* *} \rightarrow Y^{* *}$ is the biconjugate of a linear operator $T: X \rightarrow Y$, then $T^{* *}\left(X_{s}\right) \subset Y_{s}$.

The proof is trivial. Let $\left(5\right.$ be the class of Banach spaces $X$ such that $X_{s}=X^{* *}$. A. Grothendieck $[14$, p. 168$]$ has proved that $\mathfrak{P} \subset \mathbb{G}$.

Proposition 7. If $T: X \rightarrow Y$ is onto and $X$ belongs to $\mathfrak{5}$, so does $Y$. In particular, if $Z \subset X$ and $X \in \mathfrak{G}$, then $X / Z \in \mathfrak{G}$. If $C(S) \in \mathbb{G}$ and $S_{0} \subset S$, then $C\left(S_{0}\right) \in \mathbb{G}$ as $C\left(S_{0}\right)=C(S) / C\left(S \mid S_{0}\right)$.

Proposition 8. Let $A$ be a $\sigma$-complete Boolean algebra and $R$ an ideal in $A$. Let $S$ be the Stone space of the algebra $A / R$. Then $C(S) \in(5)$.

Proposition 7 follows from Lemma 11. Proposition 8 can be proved as follows. Let $S_{1}$ be the Stone space of $A$; then $C\left(S_{1}\right) \in \mathbb{5}$ can be proved analogously to the original proof of Grothendieck, i.e., applying a theorem of Banach [3, p. 222] and reducing the question to a lemma of Phillips [29, p. 525]. Since the Stone space $S$ of the quotient $A / R$ is homeomorphic to a closed subset of $S_{1}$, $C(S) \in \mathbb{G}$ follows from Proposition 7 .

There are complete Boolean algebras $A$ with $A / R$ being not $\sigma$-complete. E.g., $\beta N \backslash N$ is the Stone space of the algebra of subsets of $N$ modulo finite subsets, which is not $\sigma$-complete (Sierpiński [34]), but $C(\beta N \backslash N)=m / c_{0} \in \mathbb{6}$.

PRoblem 5. Do $C\left(\beta N_{\alpha} \backslash N_{\alpha}\right)$ belong to $\mathfrak{P}$ ?

Pełczyński and Sudakov [28] have proved that if $S$ is the Stone space of the algebra of all countable subsets of an uncountable set and their complements, then $C(S) \notin \mathfrak{P}$ though $C(S) \in \mathfrak{G}\left({ }^{15}\right)$.

By a theorem of Banach [3, p. 124] and Dixmier [8, p. 1060], if $X$ is separable, then $X_{s}$ is just the canonical image of $X$ in $X^{* *}$. This is the way Grothendieck [14] has proved that any separable injective Banach space must be finite dimensional (for another proof, see $[25$, p. 222]).

(15) J. Lindenstrauss has pointed out that a space $X^{*}$ considered in $\S 3$ of [21] does not belong to $\mathfrak{P}$ though there exists a linear operation from $C(\beta N)$ onto $X^{*}$ (whence $X^{*} \in(\mathfrak{G}$ ). Another example is due to A. Pełczyński: If $S$ contains a perfect subset, then the dual of $C(S)$ contains isomorphically the space $L\left[27\right.$, p. 214] and $L$ contains $L^{2}$ (spanned on Rademacher functions). Considering an adjoint operation, we get a linear operation from $C(S)$ onto $L^{2}$. Conversely, if $C(S)$ can be mapped onto $L^{2}$, then $S$ contains a perfect set). 
Proposition 9. Let $C(S) \in(5$. Then no infinite closed subset of $S$ is metrisable. In particular, the only $G_{\delta}$-points of $S$ are the isolated ones and the derivative of $S$ is dense-in-itself.

Problem 6. Characterize topologically those Borel subsets $A$ of $S$ such that the functional $x^{* *}(\mu)=\mu(A)$ (defined for all Radon measures on $S$ ) belongs to $X_{s}$ where $X=C(S)$, i.e., such that $\int x d \mu_{n} \rightarrow 0$ for all $x \in C(S)$ implies $\mu_{n}(A) \rightarrow 0$.

There are some sufficient conditions; e.g., if $A$ is closed and every $G_{\delta}$-set containing $A$ is a neighborhood of $A$, then $A$ has this property (cf. [32]).

Problem 7. Let $X \in \mathfrak{P}_{1}$ and be infinite dimensional. Is $X$ isomorphic to its Cartesian square?

This problem is due to A. Pełczyński (cf. [26]). There exists a compact space $S$ such that $C(S)$ is not isomorphic to its square (cf. [32]).

We conclude with two examples of very annoying problems which always arise in a field where so little is known as in this field.

Problem 8. Let $X \in \mathfrak{P}$ and be infinite dimensional. Is $X$ isomorphic to any of its hyperplanes (equivalently, is $X$ isomorphic to $X \times R, R$ being the space of reals)?

If $X \in \mathfrak{P}_{1}$, then $X$ contains a subspace isometric to $m=C(\beta N)$ whence $X \sim m \times X / m \sim m \times m \times X / m \sim m \times X$ and similarly $X \sim X \times m \sim X \times m$ $\times R \sim X \times R$, where $\sim$ means isomorphism (cf. [25]). On the other hand, if $X \in \mathfrak{P}$ and $X_{1}$ is a hyperplane in $X$, then $X_{1} \in \mathfrak{P}$; suppose that $X \sim X^{2}$ and $X_{1} \sim X_{1}^{2}$, then $X \sim X^{2} \sim\left(X_{1} \times R\right)^{2} \sim X_{1}^{2} \times R^{2} \sim X_{1} \times R^{2} \sim X \times R$.

PROBLEM 9. Does there exist a connected $S$ such that $C(S) \in(5$ ?

Amir's results $\left({ }^{10}\right)$ show that such $C(S)$ cannot be injective.

\section{REFERENCES}

1. A. Alexiewicz and Z. Semadeni, Some properties of two-norm spaces and a characterization of reflexivity of Banach spaces, Studia Math. 19 (1960), 115-133.

2. N. Aronszajn and P. Panitchpakdi, Extension of uniformly continuous transformations and hyperconvex metric spaces, Pacific J. Math. 6 (1956), 405-439.

3. S. Banach, Théorie des opérations linéaires, Monogr. Mat., Vol. 1, Warsaw, 1932.

4. F. Bohnenblust, Convex regions and projections in Minkowski spaces, Ann. of Math. (2) 39 (1938), 301-308.

5. K. Borsuk, Über Isomorphie der Funktionalräume, Bull. Internat. Acad. Polon. Sci. Let. (1933), 1-10.

6. M. M. Day, Normed linear spaces, Ergebnisse Math., Vol. 21, Springer, Berlin, 1958.

7. R. P. Dilworth, The normal completion of the lattice of continuous functions, Trans. Amer. Math. Soc. 68 (1950), 427-438.

8. J. Dixmier, Sur un théoreme de Banach, Duke Math. J. 15 (1948), 1057-1071.

9. - Sur certains espaces considérés par M. H. Stone, Summa Brasil. Math. 2 (1951), 151-182.

10. J. Dugundji, An extension of Tietze's theorem, Pacific J. Math. 1 (1951), 353-367.

11. R. E. Fullerton, Geometrical characterization of certain function spaces, Proc. Internat. Sympos. on Linear Spaces, Jerusalem, 1961, pp. 227-236.

12. A. M. Gleason, Projective topological spaces, Illinois J. Math. 2 (1958), 482-489. 
13. D. A. Goodner, Projections in normed linear spaces, Trans. Amer. Math. Soc. 69 (1950), 89-108.

14. A. Grothendieck, Sur les applications linéaires faiblement compact d'espaces du type $C(K)$, Canad. J. Math. 5 (1953), 129-173.

15. Une characterization vectorielle métrique des espaces $L^{1}$, Canad. J. Math. 7 (1955), 552-561.

16. B. Grünbaum, Some applications of expansion constants, Pacific J. Math. 10 (1960), 193-201.

17. —_ Projection constants, Trans. Amer. Math. Soc. 95 (1960), 451-465.

18. J. L. Kelley, Banach spaces with the extension property, Trans. Amer. Math. Soc. 72 (1952), 323-326.

19. C. Kuratowski, Topologie. I, Monogr. Mat., Vol. 20, Warsaw, 1948.

20. J. Lindenstrauss, On the extension property for compact operators (to appear).

21. - - A subspace of $l^{1}$ which is not isomorphic to $l^{1}$, Bull. Res. Council Israel Sect. F, (to appear).

22. L. Nachbin, A theorem of Hahn-Banach type for linear transformations, Trans. Amer. Math. Soc. 68 (1950), 28-46.

23. - Some problems in extending and lifting continuous linear transformations, Proc. Internat. Sympos. on Linear Spaces, Jerusalem, 1961, pp. 340-350.

24. H. Nakano, Über das System aller stetigen Funktionen auf einem topologischen Raum, Proc. Imp. Acad. Tokyo 17 (1941), 308-310.

25. A. Pelczyński, Projections in certain Banach spaces, Studia Math. 19 (1960), 209-228.

26. - On the isomorphism of the space $m$ and M, Bull. Acad. Polon. Sci. 6 (1958), 695-696.

27. A. Pelczyński and Z. Semadeni, Spaces of continuous functions. III, Studia Math. 18 (1959), 211-222.

28. A. Pełczyński and V. N. Sudakov, Remark on non-complemented subspaces of the space $m(S)$, Colloq. Math. 19 (1962), 85-88.

29. R. S. Phillips, On linear transformations, Trans Amer. Math. Soc. 48 (1940), 516-541.

30. J. Rainwater, A note on projective resolutions, Proc. Amer. Math. Soc. 10 (1959), 734-735.

31. W. Rudin, Averages of continuous functions on compact spaces, Duke Math. J. 25 (1958) 797-204.

32. Z. Semadeni, Banach spaces non-isomorphic to their Cartesian squares. II, Bull. Acad. Polon. Sci. 8 (1960), 81-84.

33. - Functions with sets of points of discontinuity belonging to a fixed ideal, Fund. Math. (to appear).

34. W. Sierpiński, Sur les ensembles presque contenus les uns dans les autres, Fund. Math. 35 (1948), 141-150.

35. R. Sikorski, On the representation of Boolean algebras as fields of sets, Fund. Math. 35 (1948), 247-256.

36. _- Boolean Algebras, Ergebnisse Math., Vol. 25, Springer, Berlin, 1960.

37. A. Sobczyk, On the extension of linear transformations, Trans. Amer. Math. Soc.55(1944), 153-169.

38. M. H. Stone, Algebraic characterization of special Boolean rings, Fund. Math. 29 (1937), 223-303.

39. - Boundedness properties in function lattices, Canad. J. Math. 1 (1949), 176-186.

40. J. Wada, Stonian spaces and the second conjugate spaces of AM-spaces, Osaka Math. J. 9 (1957), 195-200.

UNIVERSITY OF WASHINGTON,

Seattle, Washington

AdAM Mickiewicz UNIVERSITY, Poznan, Poland 